\title{
ERRATUM
}

Venkata Suresh Guthikonda • Ryan S. Elliott

\section{Erratum to: An effective interaction potential model for the shape memory alloy AuCd}

Received: 19 March 2010 / Accepted: 10 May 2010 / Published online: 24 November 2010

(C) Springer-Verlag 2010

\section{Erratum to: Continuum Mech. Thermodyn. DOI:10.1007/s00161-009-0109-1}

\section{Preamble}

This Erratum concerns our previous work published in this journal as: Guthikonda and Elliott (2009) 21: 269-295; DOI:10.1007/s00161-009-0109-1. While recently extending our work, we discovered a coding error in the calculation of $\frac{\partial^{2} \widetilde{\widetilde{W}}}{\partial \theta^{2}}$. This error affects the calculation of the heat capacities at constant volume, $C_{\mathrm{v}}$ (Eq. (20)), and constant pressure, $C_{\mathrm{p}}$ (Eq. (21)). However, due to the nature of the particular bug found in the computer code, only computations where multiple types of pair interactions (for example, the AuCd computations) are affected. The purpose of this erratum, therefore, is twofold: (1) to present corrected results corresponding to the model parameters as given in the original manuscript and (2) to report the results for an updated set of model parameter obtained by performing the fitting procedure described in the original manuscript with the corrected numerical code.

\section{Corrected results for original parameters}

Correcting the coding error and regenerating the results for the parameters given in Table 5 results in three corrections to the original manuscript. First, the fitted value of $C_{\mathrm{p}}$ in Table 4 changes from 26.1109 to $6.75 \mathrm{~J} \mathrm{~mol}^{-1} \mathrm{~K}^{-1}$. Second, the variation of heat capacity per mole at constant pressure $C_{\mathrm{p}}$ of B2 $\mathrm{Au}-47.5 \mathrm{at} \% \mathrm{Cd}$ versus non-dimensional temperature $\theta$ originally given in Fig. 8 now has the form shown in Fig. 16. Finally, the variation of heat capacity at constant pressure $C_{\mathrm{p}}$ of the $\mathrm{B} 2, \mathrm{~L} 1_{0}, \mathrm{~B} 19$, and $\alpha \mathrm{IrV}$ crystal structures for $\mathrm{Au}-47.5 \mathrm{at} \% \mathrm{Cd}$ with respect to non-dimensional temperature $\theta$ originally given in Fig. 15 now has the form shown in Fig. 17. The corrected $C_{\mathrm{p}}$ curves show that the original model parameters result in a B19 phase that has a negative $C_{\mathrm{p}}$ for temperatures between $\theta=1.31$ and 2.18. This indicates that this $\mathrm{B} 19$ phase is thermodynamically unstable for these temperatures. As a result, the discussion and conclusions presented in the original manuscript no longer apply for the model with the original set of parameters. However, as will be seen in Sect. 3, they do apply to the model when an updated set of parameters is used.

The online version of the original article can be found under doi:10.1007/s00161-009-0109-1.

V. S. Guthikonda · R. S. Elliott $(\varangle)$

Department of Aerospace Engineering and Mechanics, The University of Minnesota, Minneapolis, MN 55455, USA

E-mail: elliott@aem.umn.edu 


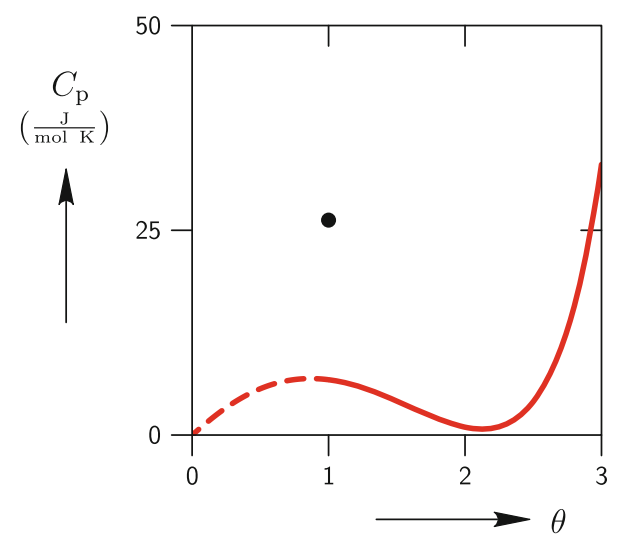

Fig. 16 Corrected variation of heat capacity per mole at constant pressure $C_{\mathrm{p}}$ of B2 $\mathrm{Au}-47.5 \mathrm{at} \% \mathrm{Cd}$ versus non-dimensional temperature $\theta$ for the parameters in Table 5. Solid circle identifies the corresponding experimental value. Solid (dashed) line indicates stable (unstable) equilibrium configurations

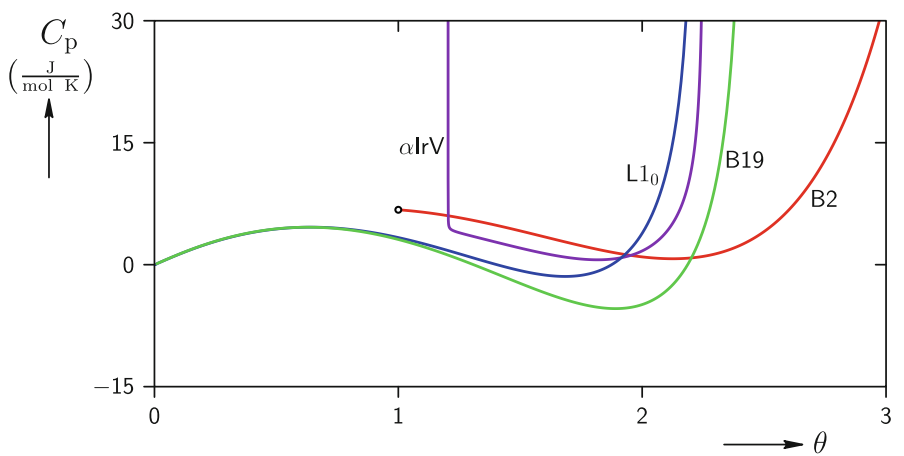

Fig. 17 Corrected variation of heat capacity at constant pressure $C_{\mathrm{p}}$ of the $\mathrm{B} 2, \mathrm{~L} 1_{0}, \mathrm{~B} 19$, and $\alpha \operatorname{IrV}$ crystal structures for $\mathrm{Au}-47.5 \mathrm{at} \% \mathrm{Cd}$ with respect to non-dimensional temperature $\theta$

\section{Updated parameter fit and results}

From Fig. 16, it is clear that the original set of potential parameters in Table 5 no longer fit $C_{\mathrm{p}}$ at $\theta=1$. Thus, an updated set of parameters for the model must be obtained by applying the originally described fitting procedure to the corrected numerical code. Accordingly, a new set of parameters for the $\mathrm{Au}-\mathrm{Cd}$ bond $\left(A_{1}^{\mathrm{ab}}, A_{2}^{\mathrm{ab}}, A_{3}^{\mathrm{ab}}, B_{1}^{\mathrm{ab}}, B_{2}^{\mathrm{ab}}, B_{3}^{\mathrm{ab}}, r_{1}^{\mathrm{ab}}, r_{2}^{\mathrm{ab}}, r_{3}^{\mathrm{ab}}\right)$ are determined by fitting $a, K$, the stability transition point of $\mathrm{B} 2 \mathrm{AuCd}, \alpha$, and $C_{\mathrm{p}}$ at the reference temperature. Additionally, $a$ is fit at multiple temperatures. Note that potential parameters corresponding to $\mathrm{Au}-\mathrm{Au}$ bond $\left(A_{1}^{\mathrm{aa}}, A_{2}^{\mathrm{aa}}, A_{3}^{\mathrm{aa}}, B_{1}^{\mathrm{aa}}, B_{2}^{\mathrm{aa}}, B_{3}^{\mathrm{aa}}, r_{1}^{\mathrm{aa}}, r_{2}^{\mathrm{aa}}, r_{3}^{\mathrm{aa}}\right)$ and $\mathrm{Cd}-\mathrm{Cd}$ bond $\left(A_{1}^{\mathrm{bb}}, A_{2}^{\mathrm{bb}}, A_{3}^{\mathrm{bb}}, B_{1}^{\mathrm{bb}}, B_{2}^{\mathrm{bb}}, B_{3}^{\mathrm{bb}}, r_{1}^{\mathrm{bb}}, r_{2}^{\mathrm{bb}}, r_{3}^{\mathrm{bb}}\right)$ remain the same as in Table 5 since the coding error did not affect the fitting results for the pure $\mathrm{Au}$ and $\mathrm{Cd}$ materials. The material properties and their fitted values for B2 AuCd are given in Table 6 and the resulting parameters are shown in Table 7.

The EIP model of Eq. (29) for the new set of parameters given in Table 7 is evaluated by generating the stress-free bifurcation diagrams Figs. 18-24 corresponding to Figs. 9-15 in Sect. 5.2 of the original manuscript. For clarity, the results for the updated parameter set are presented with a complete discussion following that given originally in Sect. 5.2.

Figure 18 displays the results by plotting the maximum principal stretch $\lambda_{3}$ versus non-dimensional temperature $\theta$. Here, the maximum principal stretch $\lambda_{3}$ is calculated as the (algebraically) largest eigenvalue of the symmetric right stretch tensor $\mathbf{U}$. Figure 18 also shows the crystal structures of the different phases that are found. Here, solid lines represent stable (CB and phonon) equilibrium configurations and dashed lines represent unstable configurations. Bifurcation points are represented by hollow circles, whereas turning points are represented by solid circles. The B2 austenite phase (red line) is stable at higher temperatures and becomes unstable as the temperature is decreased with bifurcation at $\theta=1\left(T_{\text {ref }}=333.15 \mathrm{~K}\right)$ which is in correspondence with experimental observations [1]. The onset of instability is associated with a deformation mode where the 
Table 6 Experimental values and updated model values of lattice parameter $a$ at 700, 550, 333.15, 150, and $0 \mathrm{~K}$; cohesive energy $E_{\mathrm{c}}$, bulk modulus $K$, thermal expansion coefficient $\alpha$, and heat capacity at constant pressure $C_{\mathrm{p}}$ at reference temperature $333.15 \mathrm{~K}$ $(\theta=1)$ of $\mathrm{B} 2 \mathrm{AuCd}[1,4,5,8] .{ }^{*} E_{\mathrm{c}}$ at $333.15 \mathrm{~K}$ is not actually fitted. See Sect. 4.2.1 for more details

\begin{tabular}{lllcc}
\hline B2 AuCd & & & & \\
\hline Property & Unit & Temperature $(\mathrm{K})$ & Experimental value & Fitted value \\
\hline Lattice parameter $(a)$ & $\AA$ & 700.00 & 3.3420 & 3.3492 \\
Lattice parameter $(a)$ & $\AA$ & 550.00 & 3.3316 & 3.3295 \\
Lattice parameter $(a)$ & $\AA$ & 333.15 & 3.3165 & 3.3105 \\
Lattice parameter $(a)$ & $\AA$ & 150.00 & 3.3038 & 3.2999 \\
Lattice parameter $(a)$ & $\AA$ & 0.00 & 3.2935 & 3.2940 \\
Cohesive energy $\left(E_{\mathrm{c}}\right)$ & $\mathrm{A}$ & 333.15 & -2.6936 & $-1.8318^{*}$ \\
Bulk modulus $(K)$ & $\mathrm{GPa}$ & 333.15 & 20.5103 & 99.4973 \\
Thermal expansion coefficient $(\alpha)$ & $10^{-6} \mathrm{~K}^{-1}$ & 333.15 & 26.1093 & 20.8990 \\
Heat capacity $\left(C_{\mathrm{p}}\right)$ & $\mathrm{J} \mathrm{mol}^{-1} \mathrm{~K}^{-1}$ & 333.15 & 26.1079 \\
\hline
\end{tabular}

Table 7 Updated fitted parameters for the Au-47.5at\%Cd Morse EIP model of Eq. (29)

\begin{tabular}{|c|c|c|}
\hline Potential parameter & & Value \\
\hline \multirow[t]{9}{*}{$\mathrm{Au}-\mathrm{Au}$} & $A_{1}^{\mathrm{aa}}$ & $0.482917351030788 \mathrm{eV}$ \\
\hline & $A_{2}^{\mathrm{aa}}$ & $-0.000855192503400 \mathrm{eV}$ \\
\hline & $A_{3}^{\mathrm{aa}}$ & 3.625177992551010 \\
\hline & $B_{1}^{\mathrm{aa}}$ & 4.708238552507823 \\
\hline & $B_{2}^{\mathrm{aa}}$ & -0.083925503055104 \\
\hline & $B_{3}^{\text {aa }}$ & 2.005750046077795 \\
\hline & $r_{1}^{\mathrm{aa}}$ & $3.035902348167637 \AA$ \\
\hline & $r_{2}^{\mathrm{aa}}$ & $0.040386678684356 \AA$ \\
\hline & $r_{3}^{\mathrm{aa}}$ & 0.951807321991185 \\
\hline \multirow[t]{9}{*}{$\mathrm{Cd}-\mathrm{Cd}$} & $A_{1}^{\mathrm{bb}}$ & $0.151331888205594 \mathrm{eV}$ \\
\hline & $A_{2}^{\mathrm{bb}}$ & $0.002988464666570 \mathrm{eV}$ \\
\hline & $A_{3}^{\mathrm{bb}}$ & 2.080803415890498 \\
\hline & $B_{1}^{\mathrm{bb}}$ & 4.631077953243956 \\
\hline & $B_{2}^{\mathrm{bb}}$ & -0.048032181489227 \\
\hline & $B_{3}^{\mathrm{bb}}$ & 2.117843852765962 \\
\hline & $r_{1}^{\mathrm{bb}}$ & $3.144811091489227 \AA$ \\
\hline & $r_{2}^{\mathrm{bb}}$ & $0.048803776186845 \AA$ \\
\hline & $r_{3}^{\mathrm{bb}}$ & 0.784063749845215 \\
\hline \multirow[t]{9}{*}{$\mathrm{Au}-\mathrm{Cd}$} & $A_{1}^{\mathrm{ab}}$ & $0.198800688293818 \mathrm{eV}$ \\
\hline & $A_{2}^{\mathrm{ab}}$ & $0.002241110733955 \mathrm{eV}$ \\
\hline & $A_{3}^{\mathrm{ab}}$ & 2.000000020369559 \\
\hline & $B_{1}^{\mathrm{ab}}$ & 5.452742482206239 \\
\hline & $B_{2}^{\mathrm{ab}}$ & -0.007514062001064 \\
\hline & $B_{3}^{\mathrm{ab}}$ & 3.298115567103574 \\
\hline & $r_{1}^{\mathrm{ab}}$ & $3.081961402980163 \AA$ \\
\hline & $r_{2}^{\mathrm{ab}}$ & $0.028018026615333 \AA$ \\
\hline & $r_{3}^{\mathrm{ab}}$ & 0.842955022337628 \\
\hline
\end{tabular}

shifts become non-zero as discussed in [2]. A set of B19 and $\alpha \operatorname{IrV}$ orthorhombic equilibrium paths emerge from the bifurcation point at $\theta=1$. The B19 equilibrium path (green line) is initially unstable as it emerges from the bifurcation point, but becomes stable at a turning point near $\theta=2.61$. It continues to be stable as temperature is decreased to $0 \mathrm{~K}$. It is also observed that an initially unstable path of a monoclinic structure with space group P2/m (yellow line) originates from the secondary bifurcation point on the B19 path at around $\theta=2.52$ and it continues to be unstable as temperature decreases to $0 \mathrm{~K}$. The $\alpha \mathrm{IrV}$ equilibrium path (purple 


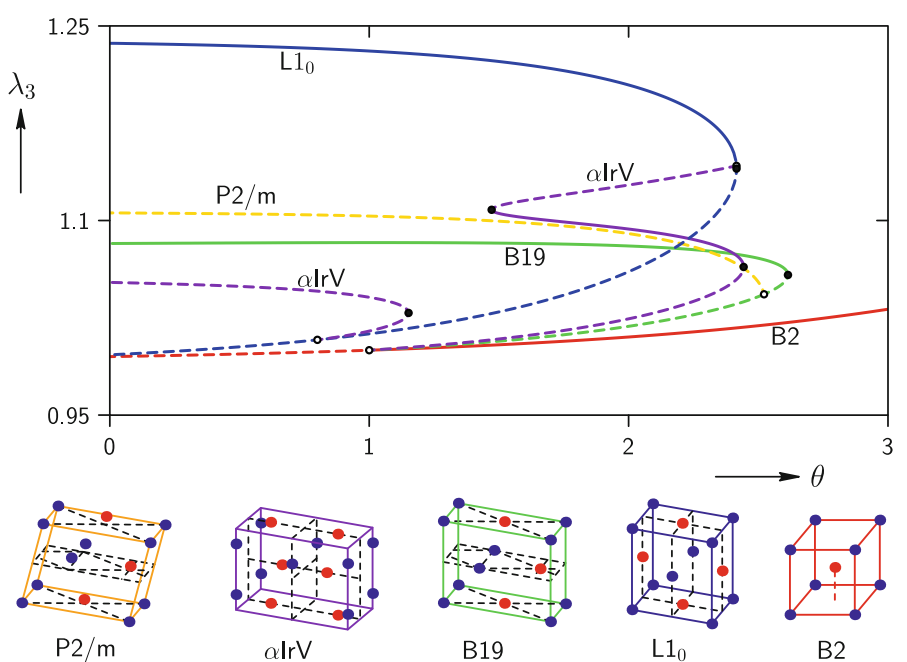

Fig. 18 Variation of $\lambda_{3}$ (largest eigenvalue of $\mathbf{U}$ ) of $\mathrm{B} 2, \mathrm{~L} 1_{0}, \mathrm{~B} 19, \alpha \mathrm{IrV}$, and $\mathrm{P} 2 / \mathrm{m}$ crystal structures of Au- $47.5 \mathrm{at} \% \mathrm{Cd}$ with respect to non-dimensional temperature $\theta$

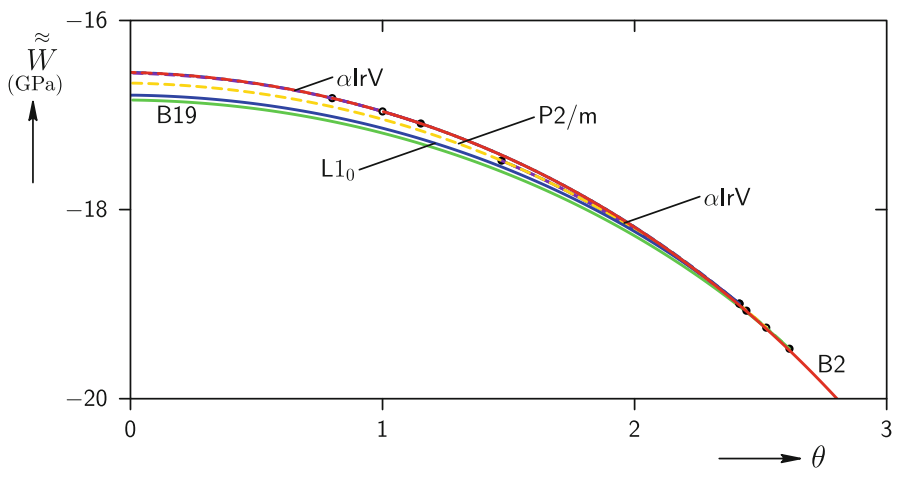

Fig. 19 Variation of (Gibbs) free energy density $\underset{W}{W}$ of $\mathrm{B} 2, \mathrm{~L} 1_{0}, \mathrm{~B} 19, \alpha \mathrm{IrV}$, and $\mathrm{P} 2 / \mathrm{m}$ crystal structures of Au-47.5at\% Cd with respect to non-dimensional temperature $\theta$

line) is initially unstable as it emerges from the B2 bifurcation point, but becomes stable at a turning point near $\theta=2.44$. It continues to be stable until $\theta=1.47$ where it encounters another turning point. The $\alpha \operatorname{IrV}$ path eventually reaches a point where it intersects an $\mathrm{L} 1_{0}$ equilibrium path at around $\theta=2.41$. Starting from this point, the $\mathrm{L} 1_{0}$ equilibrium path is found to be stable as $\lambda_{3}$ is increased until temperature reaches $0 \mathrm{~K}$. It becomes unstable as $\lambda_{3}$ is decreased and continues to be unstable through $0 \mathrm{~K}$. An additional unstable path of $\alpha \mathrm{IrV}$ is also found originating from the bifurcation point on the unstable $\mathrm{L} 1_{0}$ path at $\theta=0.80$.

The variation of the $\mathrm{HC}$ (Gibbs) free energy density $\underset{W}{W}$ with respect to the non-dimensional temperature $\theta$ is plotted in Fig. 19. The slope and curvature of all stress-free equilibrium paths are found to be negative for all temperatures. This indicates that the entropy and heat capacity at constant pressure are positive, as required by equilibrium thermodynamics.

At this point, we restrict our attention to the stable path segments for $\mathrm{B} 2, \mathrm{~L} 1_{0}, \mathrm{~B} 19$, and $\alpha \mathrm{IrV}$ predicted by the EIP model. Figure 20 displays the variation of $\widetilde{\widetilde{W}}$ of these phases with respect to $\theta$. At high temperatures the B2 crystal is the only stable phase, at intermediate temperatures four phases are stable, and at low temperatures two phases are stable. Figure 21 displays the variation of unit cell volume with respect to non-dimensional temperature $\theta$. The unit cell volumes of all stable phases are nearly equal which shows that any MTs predicted by this model will result in a volume change of less than $0.1 \%$. The unit cell volume of different phases increase as temperature is increased which is in coincidence with general experimental observations. Figure 22 displays the variation of instantaneous bulk modulus $K$ with respect to non-dimensional temperature $\theta$. For $\alpha \operatorname{IrV}$ and B19, $K \rightarrow 0$ as a turning point is approached on the respective equilibrium paths. Figures 23 and 24 display the variation of entropy and heat capacity at constant pressure, respectively, with respect to non-dimensional 


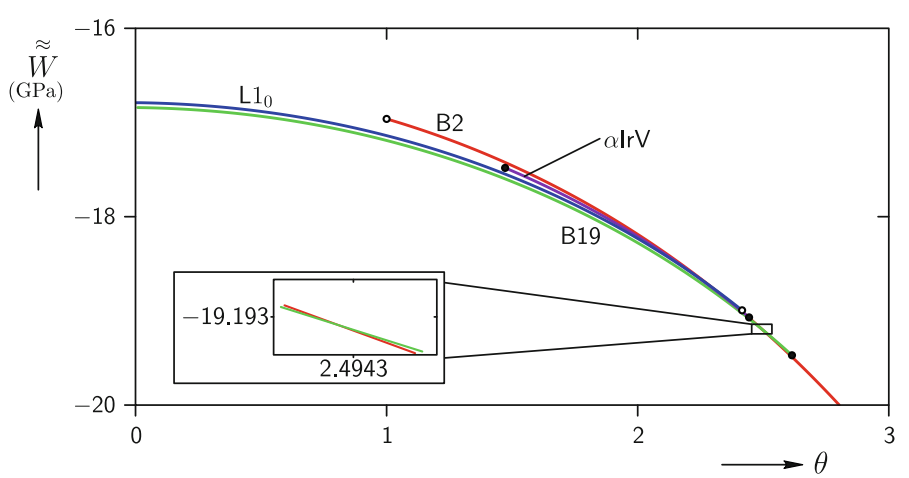

Fig. 20 Variation of (Gibbs) free energy density $\underset{W}{W}$ of stable path segments for the $\mathrm{B} 2, \mathrm{~L} 1_{0}, \mathrm{~B} 19$, and $\alpha \operatorname{IrV}$ crystal structures of Au-47.5at\%Cd with respect to non-dimensional temperature $\theta$

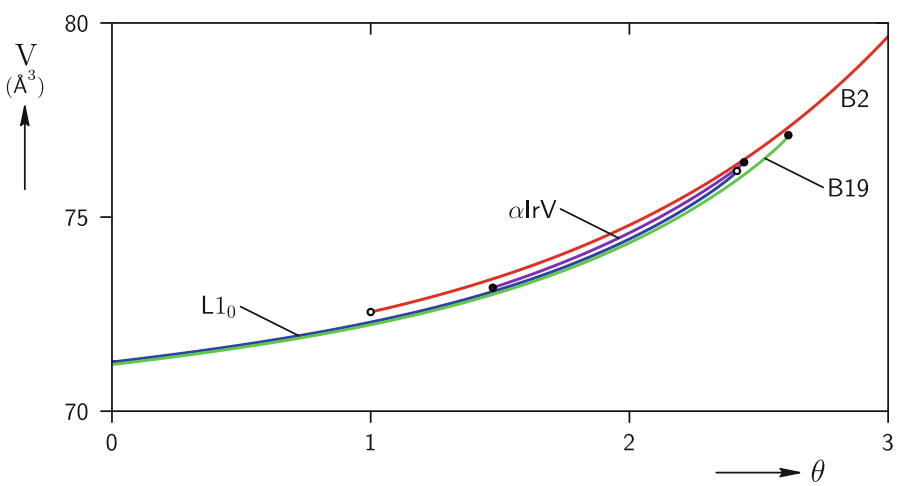

Fig. 21 Variation of unit cell volume of the $\mathrm{B} 2, \mathrm{~L}_{0}, \mathrm{~B} 19$, and $\alpha \mathrm{IrV}$ crystal structures for $\mathrm{Au}-47.5$ at $\% \mathrm{Cd}$ with respect to nondimensional temperature $\theta$

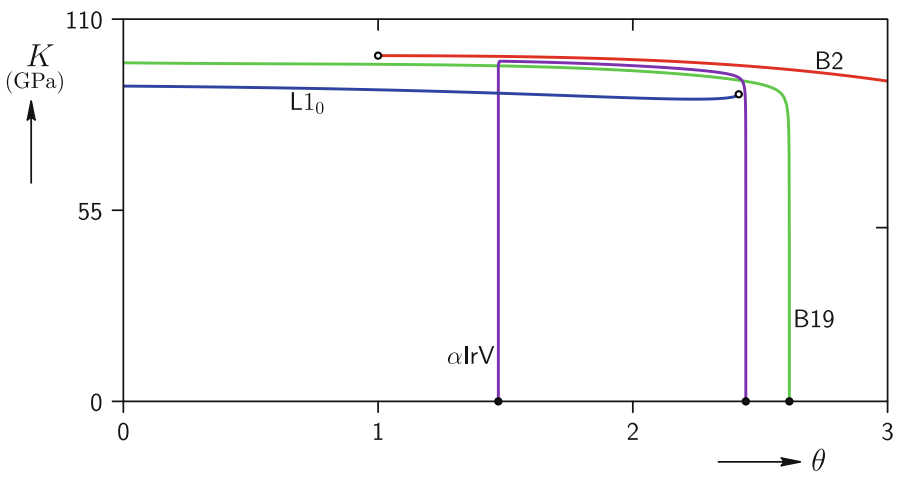

Fig. 22 Variation of instantaneous bulk modulus $K$ of the $\mathrm{B} 2, \mathrm{~L} 1_{0}, \mathrm{~B} 19$, and $\alpha \mathrm{IrV}$ crystal structures for Au-47.5at\% $\mathrm{Cd}$ with respect to non-dimensional temperature $\theta$

temperature $\theta$. In general, entropy and heat capacity are found to increase with temperature. This is in general agreement with thermodynamic theory. For the $\mathrm{L} 1_{0}, \mathrm{~B} 19$, and $\alpha \mathrm{IrV}$ phases, $C_{\mathrm{p}}$ diverges to infinity as a turning point is approached on the respective equilibrium paths. This is a result of the fact that the bulk modulus goes to zero at these points.

Returning to Fig. 20, it is observed that upon decreasing temperature the B2 phase becomes metastable at the "equilibrium thermodynamics" transition temperature $\theta=2.49$ (with the B19 phase having lower free energy for lower temperatures) and finally loses material stability at $\theta=1$ which is the models value for $A_{f}$. At this temperature, the only stable phases are B19 and $\mathrm{L} 1_{0}$. However, B19 is the stable phase with minimum free energy. Thus, a transformation from B2 to B19 can be expected. For lower temperatures, the B19 phase continues to be the stable phase with minimum free energy. In the case of increasing temperature, the 


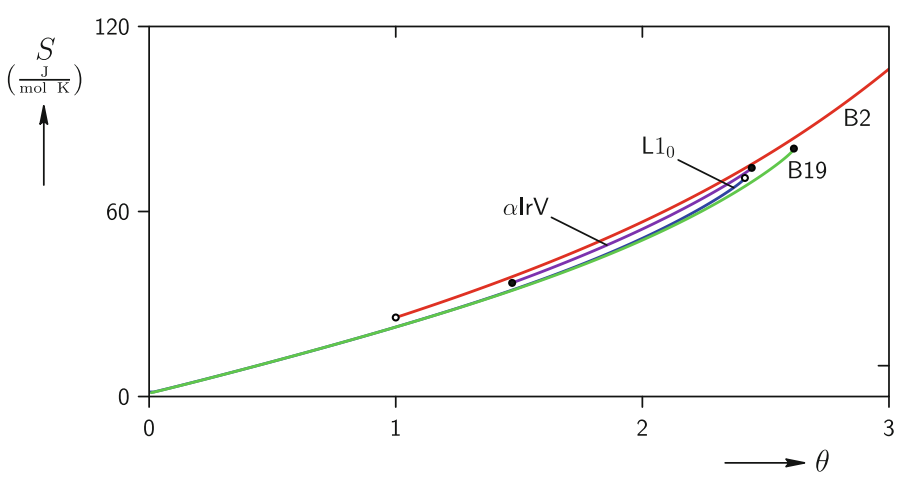

Fig. 23 Variation of entropy $S$ of the B2, $1_{0}$, B 19, and $\alpha \mathrm{IrV}$ crystal structures for Au-47.5at $\% \mathrm{Cd}$ with respect to non-dimensional temperature $\theta$

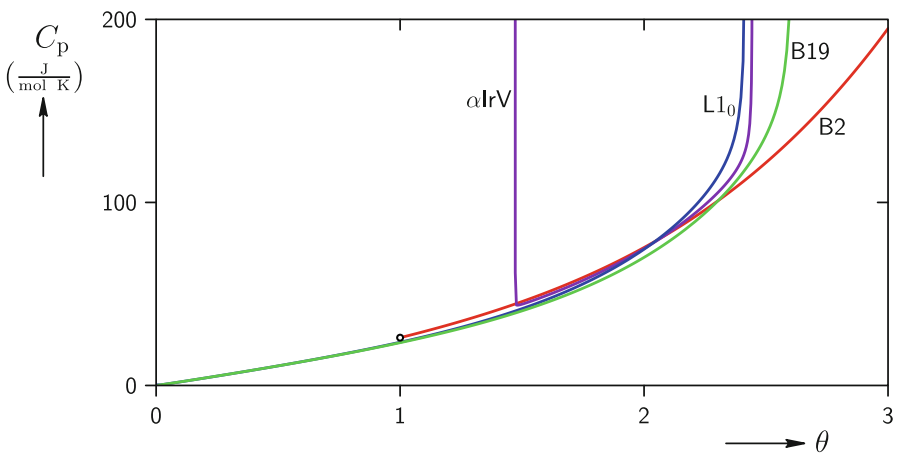

Fig. 24 Variation of heat capacity at constant pressure $C_{\mathrm{p}}$ of the $\mathrm{B} 2, \mathrm{~L} 1_{0}, \mathrm{~B} 19$, and $\alpha \mathrm{IrV}$ crystal structures for Au- $47.5 \mathrm{at} \% \mathrm{Cd}$ with respect to non-dimensional temperature $\theta$

orthorhombic B19 structure has the global minimum free energy until $\theta=2.49$ where it becomes metastable with respect to the B2 phase. It loses material stability soon afterward at $\theta=2.55$ which is the model's prediction for $M_{f}$ where it ceases to exist for higher temperatures. Ideally, one should verify that no other equilibrium crystal structures exist with lower energy. This would ensure that the minimum energy phases shown in Fig. 20 capture the model's ground state phase at any given temperature. However, this theoretically infinite investigation is not pursued in this work.

The above discussion shows that the model predicts a hysteretic martensitic transformation between the experimentally observed austenite (B2) and martensite (B19) phases for AuCd. Upon cooling the austenite to martensite, transition begins at $\theta=2.49$ and would complete at $\theta=1.0$. The reverse transformation, upon heating, would begin at $\theta=2.49$ and complete at $\theta=2.55$. Therefore, the predicted maximum possible temperature hysteresis is $\theta_{M_{f}}-\theta_{A_{f}}=1.55(516.38 \mathrm{~K})$. This is within an order of magnitude of the experimental value $30 K[1]$.

At the transformation temperature $\left(A_{f}\right), 333.15 \mathrm{~K}$ [1], the transformation stretch associated with the B2 to B19 MT is found to be

$$
\mathbf{U}^{*}=\left(\begin{array}{ccc}
1.05388872 & 0.02908900 & 0 \\
0.02908900 & 1.05388872 & 0 \\
0 & 0 & 0.89693725
\end{array}\right),
$$

where $\mathbf{U}_{\mathrm{B} 19}=\mathbf{U}^{*} \cdot \mathbf{U}_{\mathrm{B} 2}$. Here, $\mathbf{U}_{\mathrm{B} 19}$ is the right stretch tensor for the stress-free configuration of the $\mathrm{B} 19$ orthorhombic structure at the reference temperature $(\theta=1)$, and $\mathbf{U}_{\mathrm{B} 2}(=\mathbf{I})$ is the right stretch tensor of the stress-free $\mathrm{B} 2$ cubic structure at $\theta=1$. The components of $\mathbf{U}^{*}$ are given in an ortho-normal basis aligned with the reference B2 cubic axes. The corresponding experimental value [7] is

$$
\mathbf{U}_{\exp }^{*}=\left(\begin{array}{ccc}
1.0260 & 0.0100 & 0 \\
0.0100 & 1.0260 & 0 \\
0 & 0 & 0.9501
\end{array}\right) .
$$


There is reasonable agreement between the EIP model's prediction and the experimentally observed value of the transformation stretch tensor.

The latent heat $(\Delta \widetilde{W}+T \Delta S)$ associated with the B2 to B19 transformation at $\theta=1$ is calculated as $-844.5115 \mathrm{cal} \mathrm{mol}^{-1}$ (negative values correspond to exothermic transition) which is within a factor of 10 of the experimental value for the latent heat of transformation from B2 to B19 which is $-88 \mathrm{cal} \mathrm{mol}^{-1}$ [6].

Thus, it is found that the current Morse EIP model for $\mathrm{Au}-47.5 \mathrm{at} \% \mathrm{Cd}$ is able to match (by construction) the lattice parameter, instantaneous bulk modulus, and stability range of the B2 austenite phase as well as the austenite's linear thermal expansion coefficient, its heat capacity at constant pressure at $\theta=1$, and its lattice parameter at $T=550 \mathrm{~K}, 700 \mathrm{~K}$. Further, the model predicts the existence of a temperature-induced hysteretic B2 to B19 martensitic phase transformation, and the transformation stretch tensor is predicted with reasonable accuracy. Finally, the latent heat of transformation and the size of the temperature hysteresis are predicted to be within an order of magnitude.

\section{Conclusion}

After fixing the coding error in our numerical simulation code and obtaining an updated fit of our model (Table 7), the model's predictions are found to fully support the discussion and conclusions contained in Sect. 6 of original manuscript.

Acknowledgments The authors would like to acknowledge the continuing support of the National Science Foundation CAREER Grant CMMI-0746628 (Dr. Shih-Chi Liu, Program Director) and the Minnesota Supercomputing Institute.

\section{References}

1. Chang, L.C., Read, T.A.: Plastic deformation and diffusionless phase changes in metals-the AuCd $\beta$-phase. Trans. Am. Inst. Min. Metall. Eng. 191(1), 47-52 (1951)

2. Guthikonda, V.S., Elliott, R.S.: Stability and elastic properties of the stress-free B2 (CsCl-type) crystal for the Morse pair potential model. J. Elast. 92, 151-186 (2008)

3. Guthikonda, V.S., Elliott, R.S.: An effective interaction potential model for the shape memory alloy AuCd. Contin. Mech. Thermodyn. 21, 269-295 (2009)

4. Hultgren, R., Desai, P.D., Hawkins, D.T., Gleiser, M., Kelley, K.K.: Selected Values of the Thermodynamic Properties of Metals and Alloys. Wiley, New York (1963)

5. Hultgren, R., Desai, P.D., Hawkins, D.T., Gleiser, M., Kelley, K.K.: Selected Values of the Thermodynamic Properties of Binary Alloys. Wiley, New York (1973)

6. Nakanishi, N., Mori, T., Miura, S., Murakami, Y., Kachi, S.: Pseudoelasticity in Au-Cd thermoelastic martensite. Philos. Mag. 28, 277-292 (1973)

7. Ohba, T., Emura, Y., Miyazaki, S., Otsuka, K.: Crystal structure of $\gamma_{2}^{\prime}$ martensite in Au-47.5\%Cd alloy. Mater. Trans. JIM 31(1), 12-17 (1990)

8. Zirinsky, S.: The temperature dependence of the elastic constants of Gold-Cadmium alloys. Acta Metall. 4(2), 164-171 (1956) 